Veterin. Inst. d. Fachabteilung d. Fischerei u. Tierzucht, Univ. Hiroshima (Vorstand: Prof. Y. TSUJI) u. Anat. Inst. d. Med. Fak., Univ. Okayama

(Vorstand: Prof. M. SEKI).

\title{
Über die Veränderungen der Ultrastrukturdichte der Spermien während ihrer Reifung.
}

精子の成熟に伴う超構密度の変化について.

Shunsaku FUJII 藤 井 俊 策.

(Eingegangen am 26. Oktober 1956.)

Es ist bekannt, daß bei Säugetieren die im Hoden noch unreifen Spermien während des Transportes durch die Ausführungsgänge in den Hoden und Nebenhoden heranreifen. Die morphologischen Veränderungen der Spermien in dieser Reifungsperiode wurden bisher ziemlich häufig untersucht. So stellten z. B. WALDEYER (1906), BRAUS u. a. (1924) und KUGIMOTO u. a. (1930) fest, daß die am Halsteil des Spermiums vorliegenden Protoplasmatröpfchen während ihres Fortgehens durch den Nebenhodengang an sein Verbindungsstück herunterkommen und bald verschwinden.* Nach KUGIMOTO u. a. (1930) sind die Spermien im Nebenhodenkopf des Schweins verschieden groß, werden aber im Nebenhodenschwanz fast gleichgroß, wobei die Länge des Kopfes zunimmt, dagegen die Breite desselben abnimmt. Früher fand MEVES (1899) bei mehr gereiften Spermien des Meerschweinchens eine stärkere Krümmung des Kopfes in Kantenansicht. Neuerdings konstatierte TAKASHIMA (1952), daß im Hoden des Menschen der vordere Teil des Kopfes der unreifen Spermien für die Elektronenstrahlen mehr durchläßig ist als der der reifen Spermien. Diese morphologischen Veränderungen sind aber keineswegs klar ausgesprochen, und die Anerkennung ist nicht leicht. Auch auf gewöhnlichen färberischen Wegen ist die Unterscheidung der unreifen und reifen Spermien schwer. Es gelang mir jedoch, durch eine kombinierte Anwendung von verschiedenen Säurefarbstoffen nach dem Prinzip von MÖLLENDORFF (1924) und SEKI $(1936,1939,1954)$ Spermien in verschiedenen Reifezuständen und von verschiedener Ultrastrukturdichte in ganz anderen Farbtönen darzustellen. Dazu benutzte ich eine Mischfarbstofflösung von Pikrinsäure, Ponceau PR und Anilinblau zur Färbung.

* Bezüglich der Benennung der Abschnitte des Spermiums herrscht keine Übereinstimmung. Hier wird das Spermium in drei Abschnitte eingeteilt: Kopf, kurzen Hals und langen Schwanz. Der Schwanz besteht ferner aus dem Verbindungs-, Haupt- und Endstück. 


\section{Material und Färbungsmethode.}

Die Untersuchung wurde an den Spermien von geschlechtsreifen Tieren: 3 Exemplare von Rind, 17 von Hund, 3 von Schwein, 5 von Kaninchen, 3 von Meerschweinchen und 6 von der Maus, ohne Rücksicht auf die Brunstperiode vorgenommen, am ausführlichsten aber an denen von Hund. Es wurden den Tieren gleich nach dem Töten Stückchen des Hodens, Nebenhodenkopfes, seines Körpers und Schwanzes und der Ampulle des Samenleiters entnommen, zerkleinert und in physiologische Kochsalzlösung eingelegt. Nachdem man die Konzentration der Suspensionen der verschiedenen Spermien auf eine bestimmte Größe gebracht hatte, strich man diese auf ein und demselben Objektglas aus. Die Ausstriche wurden nach dem Trocknen mit neutralem 10\%igem Formalin 20 Minuten lang fixiert. Die Färbung vollzog sich in einer Lösung von Pikrinsäure, Ponceau PR und Anilinblau, welche früher von OKURA (1950) kombiniert angewandt wurden. Diese Farblösung war zum Zweck meiner Untersuchung am besten geeignet. Die Bereitung der Farblösung und das Verfahren der Färbung gingen folgenderweise vor sich.

1. Als Stammlösungen wurden eine 1\%ige Lösung von Pikrinsäure, Ponceau PR und Anilinblau hergestellt. Man mischte sie vor dem Gebrauch je nach den Arten der Spermien in verschiedenen Verhältnissen, dazu setzte man Essigsäure 2\%jg zu.

2. Vor der Färbung der Ausstriche wurden diese im Wasser etwa 20 Minuten lang aufbewahrt und eingeweicht, weil die trocken gewesenen Gebilde eine different dicht gebaute oberflächliche Schicht besitzten, welche mechanisch verschieden gegen Eindringen von Farbstoffen widerstehen würden, worauf SEKI (1934) bei der Bestimmung des isoelektrischen Punktes der Erythrocyten auf färberischem Wege aufmerksam gemacht hat.

3. Man füllte das Objetglas mit der Farblösung. Die Färbung dauerte bei $37^{\circ} \mathrm{C} 20-30$ Minuten lang, während deren man die Farbstofflösung von Zeit zu Zeit schaukelte.

4. Nach ein paar Sekunden langer Abspülung im Wasser brachte man die Ausstriche durch 95\%igen und absoluten Alkohol in Xylol, um sie schließlich in Kanadabalsam einzuschließen.

Andererseits wurden die Ausstriche nach der BIELSCHOWSKYschen Versilberung, der Azanmethode, der HEIDENHAINschen Eisenhämatoxylinmethode und mit 1\%igem Eosin gefärbt. Da die miteinander zu vergleichenden Ausstriche immer auf ein und demselben Objektglas angefertigt waren, wurden sie unter ganz gleichen Bedingungen fixiert, gefärbt und nachbehandelt. 


\section{Ergebnisse.}

\section{A. Spermien des Hundes.}

Zur Färbung der Hundspermien eignet sich das Gemisch von den oben beschriebenen Stammlösungen im Verhältnis von $1: 3: 3$. Die Resultate der Färbung der Spermien des Hundes aus verschiedenen Abschnitten des Samenweges waren die folgenden.

1. Spermien aus dem Hoden des Hundes.

In den Ausstrichen aus dem Hoden finden sich Samenzellen in verschiedenen Stadien der Entwickelung.

Der Kern der Präspermiden färbt sich orange, ihr Cytoplasma aber rot oder schwach violettrot, daher sind die beiden distinkt zu unterscheiden (Abb. $1_{1}$ ). In den jungen Spermiden färbt sich das Cytoplasma mit zunehmendem rotem Ton und erscheint orangerot, während der Kern noch orange färbbar ist, so daß die Differenz der Färbbarkeit der beiden sich vermindert. Das Acrosom in der Nähe des Kerns färbt sich intensiv blau (Abb. 12). Die umformten Spermiden in späteren Stadien haben einen spindelförmigen Kern, welcher braunrot färbbar ist. Das auf der kaudalen Seite des Kerns in Ellipsenform vorhandene Cytoplasma färbt sich schwach orange, der in ihm befindliche Schwanz aber mit stärkerem rotem Ton orangerot $\left(\mathrm{Abb} .1_{3}\right)$. Die vordere Hälfte des Kopfes mit der Kappe wird dabei schwach blau gefärbt.

Die Spermien in ausgebildeter Form sind bezüglich ihrer Größe und Färbbarkeit keinesweges gleich. Die Spermien im Hoden werden bezüglich ihrer Färbbarkeit in 3 Ordnungen eingeteilt. Bei den Spermien 1. Ordnung färbt sich der vordere Teil des Kopfes mit der Kappe schwach blau, und der hintere Teil desselben rot und der Schwanz orange (Abb. 14). Bei den Spermien 2. Ordnung färbt sich aber der vordere Teil des Kopfes etwas stärker blau und sein hinterer Teil und der Schwanz orange (Abb. 15). Bei den Spermien 3. Ordnung wird der vordere Teil des Kopfes noch stärker blau, der hintere Teil desselben gelb und der Schwanz rotorange (Abb. 16). Mit Heranreifung der Spermien von der 1. bis zur 3. Ordnung werden also alle Abschnitte derselben ultrastrukturell dichter. Die Spermien 1. Ordnung sind die jüngsten, und ihre Kopfform und Färbbarkeit unterscheiden sich nicht viel von denen der Spermiden in späteren Stadien. Die Spermien 2. Ordnung besitzen den Kopf, der etwas breiter und kürzer ist als bei den Spermien 1. Ordnung. Die Spermien in der Ampulle färben sich von diesen abweichend. Man nennt sie die Spermien 4. Ordnung, worauf wir später zurück kommen. Die Spermien 3. Ordnung sind doch fast ausgereift. Im Hoden kommen die Spermien 3. Ordnung weitaus weniger vor als die Spermien 1. und 2. Ordnung. Es gibt auch zahlreiche Spermien, deren Kappe nicht fest aufsitzt oder herausgefallen ist und deren vordere 
Hälfte sich mit dem gleichen Ton färbt wie die hintere Hälfte.

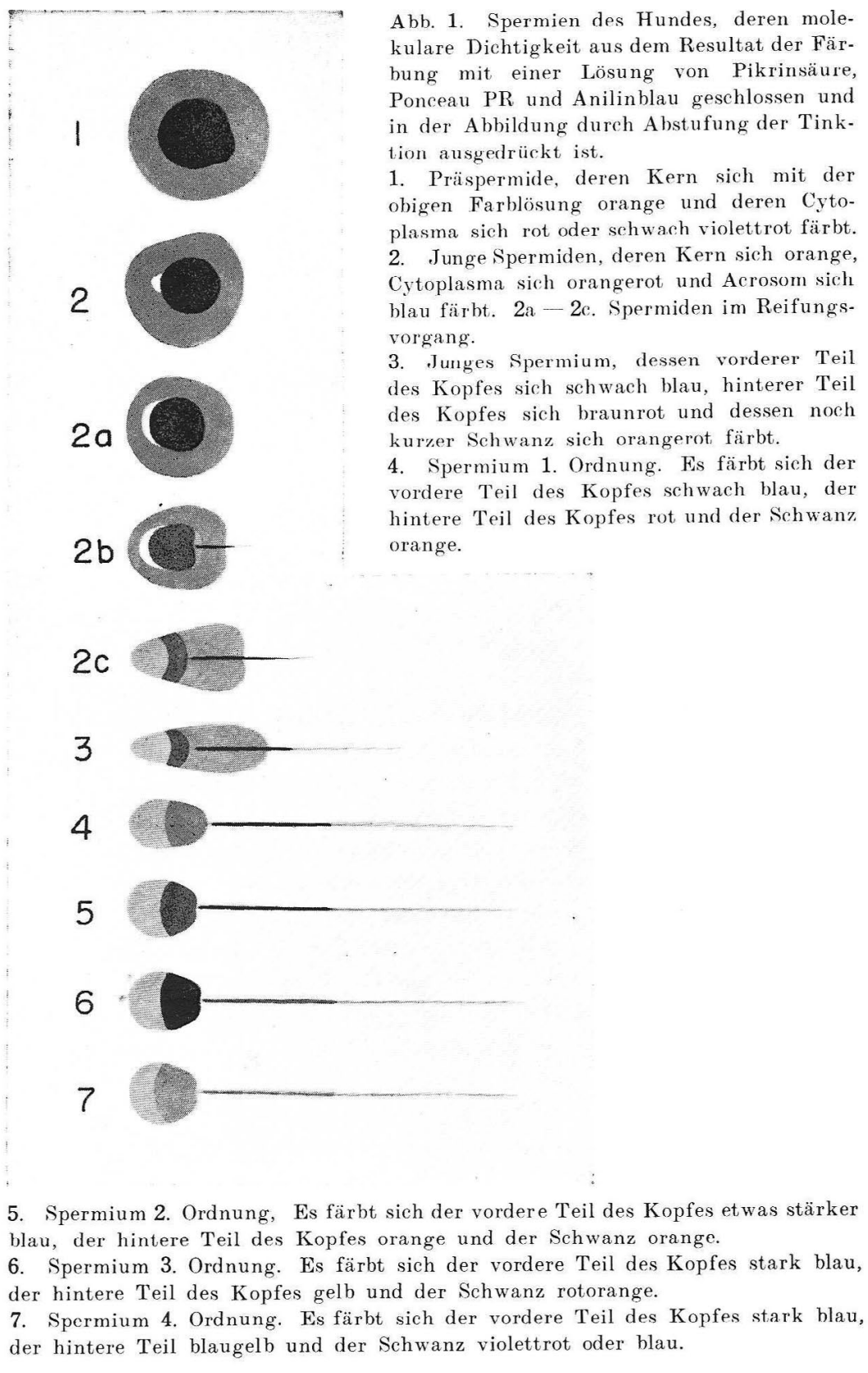

Abb. 1. Spermien des Hundes, deren molekulare Dichtigkeit aus dem Resultat der Färbung mit einer Lösung von Pikrinsäure, tion ausgedrückt ist.

1. Präspermide, deren Kern sich mit der plasma sich rot oder schwach violettrot färbt. 2. Junge Spermiden, deren Kern sich orange, Cytoplasma sich orangerot und Acrosom sich blau färbt. 2a-2c. Spermiden im Reifungsvorgang.

3. Junges Spermium, dessen vorderer Teil des Kopfes sich schwach blau, hinterer Teil des Kopfes sich braunrot und dessen noch kurzer Schwanz sich orangerot färbt.

4. Spermium 1. Ordnung. Es färbt sich der vordere Teil des Kopfes schwach blau, der hintere Teil des Kopfes rot und der Schwanz orange. 
2. Spermien aus dem Nebenhodenkopf des Hundes.

In den Ausstrichen aus dem Nebenhodenkopf sind die Spermien 3. Ordnung im Vergleich zu den Spermien 1. und 2. Ordnung immer noch weniger vorhanden. Spermien mit herausgefallener Kappe sind auch zahlreich zu sehen.

3. Spermien aus dem Nebenhodenkörper des Hundes.

Die Spermien 3. Ordnung vermehren sich auf Kosten der Spermien 2. Ordnung. Die Spermien 1. Ordnung sind beträchtlich weniger geworden.

4. Spermien aus dem Nebenhodenschwanz des Hundes.

Die am häufigsten vorkommenden Spermien sind die der 3. Ordnung. Die Spermien 2. Ordnung sind selten, und die der 1. Ordnung nicht mehr zu sehen. Sehr häufig sieht man solche Spermien, die am Verbindungsstüch blau färbbare Protoplasmatröpfchen haben.

\section{Spermien aus der Ampulle des Hundes.}

Die Spermien in den Ausstrichen aus der Ampulle sind ausschließlich solche, deren Kopf aus dem blau gefärbten vorderen Teil und dem schwach gelbblau gefärbten hinteren Teil besteht, und die den violettrot bis blau gefärbte Schwanz haben. Man nennt sie als die Spermien 4. Ordnung (Abb. 17). Im Vergleich zu den Spermien 3. Ordnung färben sich der hintere Teil des Kopfes und der Schwanz mit einem vermehrten blauen Ton.

Die soeben erklärten, nach der Färbung mit Farblösung von Pikrinsäure, Ponceau PR und Anilinblau in vier Arten eingeteilten Spermien in den verschiedenen Abschnitten des Samenweges werden in der umstehenden Tabelle in der Prozentzahl gezeigt. Dabei wurden bei jeder Aufzählung 200 Spermien in Betrachl gezogen. Obgleich die Zahlenverhältnisse in einzelnen Fällen beträchtlich verschieden sind, kann man im allgemeinen sagen, daß die Spermien 1. Ordnung am zahlreichsten im Hoden vorhanden sind, aber im Kopf des Nebenhodens etwas weniger und im Schwanz nicht mehr, daß die Spermien 2. Ordnung sich im Hoden und im Kopf des Nebenhodens am zahlreichsten, im Körper des Nebenhodens weniger und im Schwanz nicht finden, dagegen die Spermien 3. Ordnung im Hoden und im Kopf des Nebenhodens viel weniger zu finden sind als im Körper des letzteren, um dann im Schwanz des Nebenhodens ausschließlich vorzukommen.

Zum Vergleich wurden die Spermien nach der Versilberung nach BIELSCHOWSKY untersucht. Der vordere Teil des Kopfes der Spermien 1. Ordnung tingiert sich dunkelbraun und der hintere Teil schwarz, aber der Schwanz schwach dunkelbraun. Bei den Spermien 2. Ordnung wird der vordere und hintere Teil des Kopfes dunkelbraun und der Schwanz braun tingiert. Bei den Spermien 3. Ordnung färbt sich der Kopf gleichmäßig gelbbraun, und der Schwanz ebenfalls gelbbraun. Der Kopf der 
Spermien 1. bis 3. Ordnung aus den verschiedenen Abschnitten des Samenwegs des Hundes (\%).

\begin{tabular}{|c|c|c|c|c|c|c|c|c|c|c|c|c|}
\hline \multirow[b]{2}{*}{$\mathrm{Nr}$. } & \multicolumn{3}{|c|}{ Hoden } & \multicolumn{3}{|c|}{ Nebenhodenkopf } & \multicolumn{3}{|c|}{$\begin{array}{l}\text { Nebenhoden- } \\
\text { körper }\end{array}$} & \multicolumn{3}{|c|}{$\begin{array}{l}\text { Nebenhoden- } \\
\text { schwanz }\end{array}$} \\
\hline & $\begin{array}{c}1 . \\
\text { Ordn. }\end{array}$ & $\begin{array}{c}2 . \\
\text { Ordn. }\end{array}$ & $\begin{array}{c}3 . \\
\text { Ordn. }\end{array}$ & $\begin{array}{c}1 . \\
\text { Ordn. }\end{array}$ & $\begin{array}{c}2 . \\
\text { Ordn. }\end{array}$ & $\begin{array}{c}3 . \\
\text { Ordn. }\end{array}$ & $\begin{array}{c}1 . \\
\text { Ordn. }\end{array}$ & $\begin{array}{c}2 . \\
\text { Ordn. }\end{array}$ & $\mid \begin{array}{c}3 . \\
\text { Ordn. }\end{array}$ & $\begin{array}{c}1 . \\
\text { Ordn. }\end{array}$ & $\begin{array}{c}2 . \\
\text { Ordn. }\end{array}$ & $\begin{array}{c}3 . \\
\text { Ordn. }\end{array}$ \\
\hline 1 & 22.3 & 46.9 & 30.8 & 15.4 & 49.4 & 35.2 & - & - & 100 & 一 & - & 100 \\
\hline 2 & 6.5 & 25.3 & 68.2 & 3.1 & 16.8 & 80.1 & - & - & 100 & - & - & 100 \\
\hline 3 & 6.6 & 35.1 & 58.3 & 1.4 & 24.3 & 74.3 & - & - & 100 & - & 一 & 100 \\
\hline 4 & 15.2 & 43.4 & 41.4 & 3.0 & 45.6 & 51.4 & - & - & 100 & - & - & 100 \\
\hline 5 & 13.4 & 50.1 & 36.5 & 5.4 & 48.3 & 46.3 & - & 13.5 & 86.5 & - & 一 & 100 \\
\hline 6 & 12.2 & 52.6 & 35.2 & 15.8 & 39.9 & 44.3 & - & - & 100 & - & - & 100 \\
\hline 7 & 31.6 & 53.7 & 14.7 & 16.3 & 49.5 & 34.2 & - & 22.6 & 74.4 & - & - & 100 \\
\hline 8 & 13.1 & 52.3 & 34.6 & 14.3 & 51.6 & 34.1 & 2.5 & 8.4 & 89.1 & - & - & 100 \\
\hline 9 & 37.7 & 42.1 & 20.2 & 11.2 & 44.7 & 44.1 & 6.2 & 23.6 & 70.2 & - & - & 100 \\
\hline 10 & 18.4 & 50.1 & 31.5 & 4.5 & 38.3 & 57.2 & - & 22.2 & 77.8 & - & - & 100 \\
\hline 11 & 6.2 & 66.6 & 27.2 & 2.9 & 52.1 & 45.0 & - & - & 100 & - & - & 100 \\
\hline 12 & 14.1 & 32.5 & 53.4 & 3.6 & 51.1 & 45.3 & - & 13.1 & 86.9 & 一 & - & 100 \\
\hline 13 & 5.9 & 37.7 & 56.4 & 3.1 & 30.8 & 66.1 & - & - & 100 & - & - & 100 \\
\hline 14 & 15.3 & 32.6 & 52.1 & 4.2 & 29.1 & 66.8 & - & - & 100 & - & - & 100 \\
\hline 15 & 29.3 & 35.1 & 35.6 & 5.2 & 61.3 & 33.5 & - & 39.1 & 60.9 & - & - & 100 \\
\hline 16 & 23.6 & 36.2 & 40.2 & 3.6 & 59.3 & 37.1 & - & 9.4 & 90.6 & - & - & 100 \\
\hline 17 & 10.3 & 44.6 & 45.1 & 8.1 & 39.6 & 52.3 & - & - & 100 & - & - & 100 \\
\hline i. M. & 16.6 & 43.3 & 40.1 & 7.1 & 43.0 & 49.8 & 0.5 & 8.9 & 90.4 & - & - & 100 \\
\hline
\end{tabular}

Bemerkung: Es gibt noch Spermien 4. Ordnung, die aber erst in der Ampulle des Samenleiters erscheinen.

Spermien 4. Ordnung erscheint schwach dunkelbraun, und ihr Schwanz schwach gelblichbraun. Es kann also gesagt werden, daß je stärker die Spermien in unserer Farblösung mit stärkerem rotem Ton färbbar sind, um so dunkler sie nach der Versilberung imprägniert werden.

Nach der Azanmethode werden die Spermien zwar im allgemeinen mit zunehmender Reifung mit stärkerem gelblichem Ton färbbar, aber die Veränderungen der Färbbarkeit mit der Reifung erscheinen nicht so präzis wie nach der Färbung in kombinierter Anwendung von Pikrinsäure, Ponceau PR und Anilinblau.

Die HEIDENHAINsche Eisenhämatoxylinmethode zeigte schließlich eine nur schwache Färbbarkeitsdifferenz der Spermien in verschiedenen Reifezuständen.

\section{B. Spermien der Maus.}

Die Spermien der Maus in verschiedenen Reifezuständen werden mit der gleichen Farblösung, welche man bei den Hundespermien benutzte, 
gleichmäßig stark orargegelb gefärbt, ohne daß Differenzen der Färbbarkeit zwischen den reifen und unreifen Spermien auftreten. Man ersieht daraus, daß die Mäusespermien viel dichter gebaut sind als die Hundespermien. Verwendet man für die Mäusespermien ein Gemisch der Stammlösungen im Verhältnis von $3: 1: 1$, so erhält man die gleichen Resultate wie bei den Hundespermien.

\section{Spermien des Rindes.}

Für die Rindspermien erweist sich das Gemisch der Stammlösungen im Verhältnis von 1:3:2 als das beste. Bei den Rindspermien ist die Veränderungen der Färbbarkeit mit der Reifung zwar nicht so markant wie bei den Hundespermien, sie ändert sich aber auch im gleichen Sinne wie bei ihnen.

\section{Spermien des Kaninchens, Schweins und Meerschweinchens.}

Die Spermien von diesen Tieren in verschiedenen Reifezuständen färben sich, wenn man die Farblösung, die bei den Hundespermien benutzt wurde, anwendet, prinzipiell in gleicher Weise wie bei den Hundespermien.

\section{Auswertung der Ergebnisse.}

Färbt man die Spermien aus verschiedenen Abschnitten des Samenwegs mit einer gewissen Lösung von Pikrinsäure, Ponceau PR und Anilinblau, so sieht man, daß die Färbbarkeit der Spermien sich mit der Reifung verändert. Nach den früheren Untersuchungen von REDENZ (1924) und YOCHEM (1930) sollen die Spermien auf dem Wege durch den Nebenhodengang mit einer Schicht von Sekretsubstanz aus den Epithelzellen umgehüllt werden. LANZ (1929) konstatierte beim Meerschweinchen, daß pH im Hoden $7.15-7.37$ und im Nebenhoden $6.48-6.61$ beträgt. Eine solche Umhüllung der Spermien der Sekretsubstanz und die Differenz von $\mathrm{pH}$ des Mediums können nicht die Ursache der Veränderung der Färbbarkeit der Spermien sein, weil in demselben Abschnitt des Samenwegs verschieden färbbare Spermien vorhanden sind. Die Veränderung der Färbbarkeit der Spermien beruht offensichtlith auf der Veränderung der Struktur der Spermien selbst. Die Molekülgröße der hier angewandten Farbstoffen ist bei Pikrinsäure die kleinste, bei Ponceau PR mittelgroß und bei Anilinblau die größte. Die Verstärkung der rötlichen Färbbarkeit des Hinterteils des Kopfes und die der bläulichen Färbbarkeit des Schwanzes weist also auf die Veränderung der Dichtigkeit ihres molekularen Baues hin.

Diese Tatsache läßt sich durch die Resultate der Versilberung bestätigen. Wie SEKI (1940) und SUWA (1956) dargestellt haben, können ultrastrukturell mäßig locker gebaute Strukturen durch Versilberung ge- 


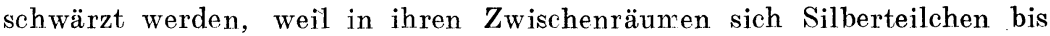
zu genügender Größe entwickeln, dagegen färben sich zu dicht gebaute Strukuren höchstens gelb bis braun, weil in ihren schmalen Gefügelücken sich solche schwarz erscheinende Silberteilchen nicht entwickeln können. Die Befunde bei der Versilberung, daß junge Spermien schwarz, herangereifte aber gelbbraun erscheinen, können also verstanden werden, wenn man annimmt, daß die Spermien während des Transportes durch den Samenweg heranreifen und ultrastrukturell dichter werden.

Es ist physiologisch von vielen Seiten nachgewiesen, daß die Spermien während des Transportes reifen. YOUNG (1930) erzielte z. B. Konzeption beim Meerschweinchen mit den Spermien aus dem Nebenhodenkopf bei

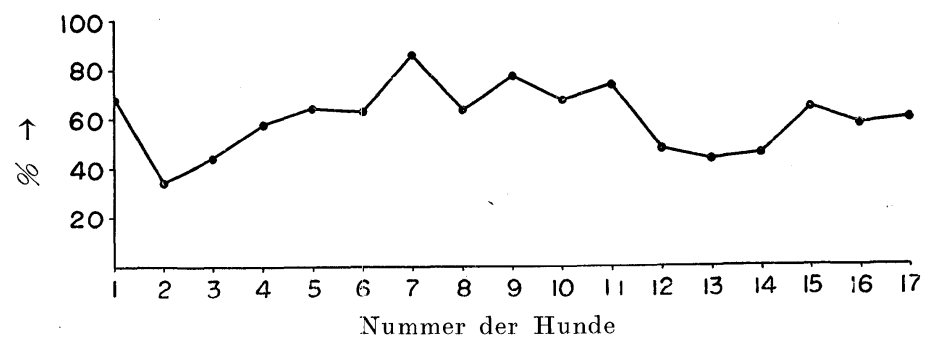

1. Unreife Spermien aus dem Hoden. 59.9\% im Mittel.

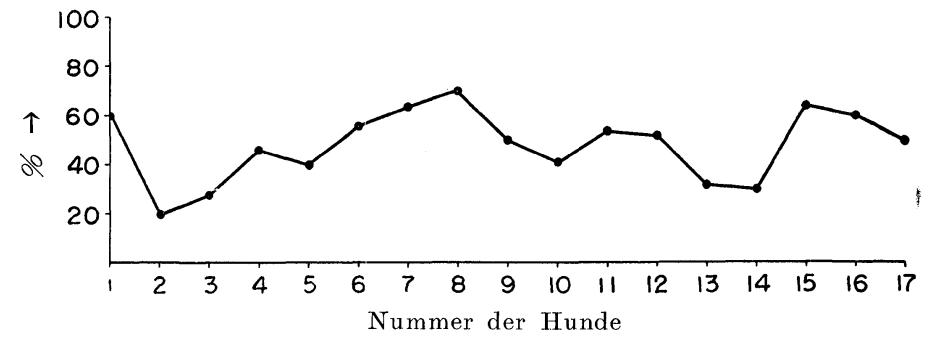

2. Unreife Spermien aus dem Nebenhodenkopf. 50.1\% im Mittel.

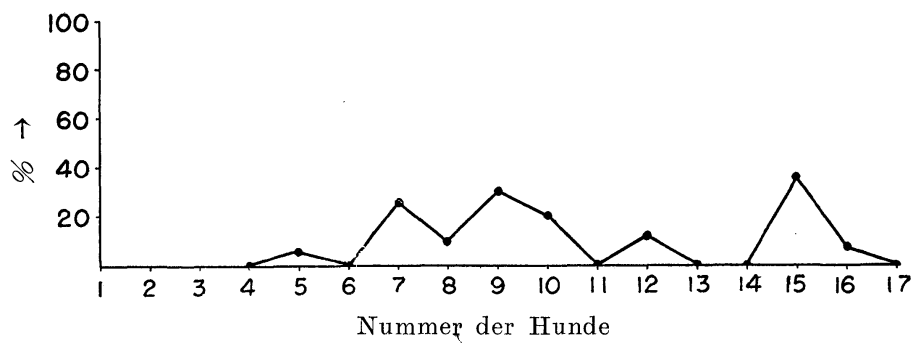

3. Unreife Spermien aus dem Nebenhodenkörper. 9.4\% im Mittel.

Abb. 2. Prozentzahl der unreifen Spermien in verschiedenen Abschnitten des Samenwegs des Hundes. 
33\% der Fälle, mit den Spermien aus dem Nebenhodenschwanz aber bei 66\%. Nach KUGIMOTO (1930) betrug die Lebensdauer der Spermien des Schweins in vitro, bezogen auf die der Spermien aus dem Nebenhodenschwanz, welche $=100$ gesetzt wurde, 70 bei den Spermien aus dem Nebenhodenkörper und 16 bei denen aus dem Nebenhodenkopf. Nach der Untersuchung von YOCHEM (1930) lebten die Rattenspermien aus dem Nebenhodenkopf 6 Tage in vitro, und die aus dem Nebenhodenschwanz 8 Tage, die aus den Samenleiter aber 7 Tage. In Abb. 1 sind die gefärbten Spermien in verschiedenen Reifezuständen schematisch dargestellt. Man bezeichnet einfachheitshalber die Spermien 1. und 2. Ordnung als die unreifen Formen, die Spermien 3. Ordnung als die reifen und stellt die Prozentzahl der unreifen Formen in verschiedenen Abschnitten des Samenwegs graphisch in Abb. 2 dar. Man ersieht daraus, daß im Hoden die unreifen Formen zu 50-60\% und die reifen zu 40-50\% vorhanden sind, im Nebenhodenkopf aber umgekehrt $40-50 \%$ bzw. bei $50-60 \%$, ferner im Nebenhodenkörper die Spermien im größten Teil die reifen Formen darstellen, und die unreifen sich weniger als zu 20\% finden, daß endlich im Nebenhodenschwanz nur die reifen Formen vorhanden sind. Damit ist bewiesen, daß die physiologischen Reifeprozesse der Spermien einfach durch das Färbungsverfahren nachgewiesen werden können.

\section{Zusammenfassung.}

Nach der Färbung der Spermien mit einer Lösung von Pikrinsäure, Ponceau PR und Anilinblau in einem bestimmten Verhältnis werden die Spermien bezüglich der Färbbarkeit in vier 4 Formen eingeteilt. Bei den Spermien 1. Ordnung färbt sich die Kopfkappe schwach blau, der hintere Teil des Kopfes rot und der Schwanz orange. Bei den Spermien 2. Ordnung wird die Kopfkappe blau gefärbt, der hintere Teil des Kopfes und der Schwanz aber orange. Bei den Spermien 3. Ordnung ist die Kopfkappe intensiv blau färbbar, der hintere Teil des Kopfes aber gelb und der Schwanz orangerot. Bei den Spermien 4. Ordnung wird die Kopfkappe blau, der hintere Teil des Kopfes blaugelb und der Schwanz violett oder blau. Während des Transportes der Spermien durch den Samenwegs nehmen diejenigen mit dem stärker gelb färbbaren Kopf und dem stärker blau färbbaren Schwanz auf Kosten derjenigen mit dem stärker rot färbbaren Kopf und schwächer orange färbbaren Schwanz an Zahl zu. Es wird also mit dem Reifungsprozess der Spermienkopf ultrastrukturell dichter und der Schwanz lockerer.

\section{内 容自 抄.}

諸種哺乳動物の精液路の諸部位からの精子の叙布を $10 \%$ 中性ホルマリン で固定し，分散度と色調の異るピクリン酸，ポンソーPR，アニリン青の一 
定濃度の混和溶液で染めると, 特飞精子頭の染色調の違う4 位の精子が区 別された. 即ち第 1 位精子では頭の前半が薄く青，後半が赤，尾が橙飞染 まり，第 2 位精子では頭の前半が青，後半が橙，尾が橙飞染まり，第 3 位 精子では頭の前半が強く青, 後半が黄, 尾が橙赤飞染まり, 第 4 位精子で は頭の前半が青，後半が青黄，尾が紫または青に染まる，殬丸から睪上体 尾飞行くに従って頭が強く赤飞, 尾が橙に染まる精子が減ずる代りに, 䫓 が強く黄飞，尾が強く青に染まる精子が増す。即ち成熟するととるに，頭 の超構密度が高くなり尾のものが低くなる。用いられた 6 種類の精子の 5 このような染色性の変化の最も著しい精子は犬のものであり, 変化の最も ち少い精子は二十日鼠のものであった。

\section{Literatur.}

Braus, H. u. E. Redenz: Nebenhoden und Samenfäden. Verh. anat. Ges. 1924. - Kugimoto, S., S. Masuda u. T. Niwa: Studien über die Spermien. Über die Vitalität und Form der Spermien im Nebenhoden des Schweins. (Jap.) Verh. jap. zootechn. Ges. 12 (1930). - Lanz, T. V.: Die reele Acidität in den einzelnen Abschnitten des männlichen Genitalapparates der Ratte und ihre hormonale Bedingtheit. Pflügers Arch. 222 (1929). - Meves, Fr.: Über die Struktur und Histogenese der Samenfäden des Meerschweinchens. Arch. mikr. Anat. 54 (1899). - Möllendorff, W. u. M.v.: Untersuchungen zur Theorie der Färbung fixierter Präparate. III. Erg. Anat. 25 (1924). - Okura, T.: Modellversuche über die Beziehung der Ultrastrukturdichte und der Färbbarkeit. (Jap. m. dtsch. Zfass.) Arch. hist. jap. 1 (1950). - Redenz, E.: Versuch einer biologischen Morphologie des Nebenhodens. Arch. mikr. Anat. 103 (1924). - Seki, M.: Studien der elektrischen Ladung und Färbbarkeit der Erythrocyten. V. Bestimmung des isoelektrischen Punktes der fixierten Erythrocyten auf färberischem Wege mit besonderer Berücksichtigung des Widerstandes der Zellmenbran gegen Eindringen von Farbstoffen. Z. ges. exp. Med. 94 (1934). - Zur physikalischen Chemie der histologischen Färbung. XI. Z. Zellforsch. 24 (1936). - Untersuchungen mit nichtwässerigen Flüssigkeiten. VIII. Z. Zellforsch. 29 (1939). - Zur Theorie der histologischen Silberschwärzung. I u. II. Z. Zellforsch. 30 (1940). - Histologische Untersuchungsmethoden und physikalische Chemie. (Jap.) Tokyo, 1954. - Suwa, K.: Vergleichende Beobachtung der Elektronenbilder der nach GÖMÖRI und BIELSCHOWSKY versilberten Kollagenfasern'und der nach GÖMÖRI und PAP versilberten Retikulumfasern. Arch. hist. jap. 10 (1956). - Takashima. T.: Elektronenmikroskopie der Menschenspermien. (Jap.) Elektronenmikr. 2 (1952). Young, W. C.: A study of the function of the epididymis. III. Functional changes undergone by spermatozoa during their passage through the epididymis and vas defferens in the guineapig. J. exper. Biol. 8 (1931). - A further study of the epididymal spermatozoon relationship in the guineapig. Anat. Rec. 44 (1929). - Yochem, D. E. : A study of the motility and resistance of rat spermatozoa at different lebels in the reproductive tract. Anat. Rec. 45 (1930). Waldeyer, W.: Die Geschlechtszellen. Hertwigs Handbuch der vergleichenden und experimentellen Entwicklungslehre der Wirbeltiere. Bd. 2. Jena, 1906. 